\title{
Preparation of Mono-Dispersed Carbon Nanotubes (CNTs) with Dodecyl Itaconate and Its Utilization in Paper-Making
}

\author{
Xiao-Shui Wang \\ Japan Chemical Analysis Center, Chiba City, Japan \\ E-mail: $x$-wang@jcac.or.jp \\ Received September 9, 2010; revised November 29, 2010; accepted December 17, 2010
}

\begin{abstract}
Dodecyl itaconate (DI) was used as the dispersant for preparation of mono-dispersed carbon nanotubes in aqueous solution in this study. It is a unique type of anionic surfactant synthesized by the authors, and its high capability for dispersing CNTs is attributed to the double bond moiety on its head-group. Furthermore, author mixed aqueous mono-dispersed CNTs with pulp to produce the CNT-based paper. Different from the reported papermaking process, a novel adjusting PH process was employed to prepare paper. This method could transfer easily dispersing mono-dispersed CNTs in aqueous onto the surfaces of cellulose fibers, because PH adjustment of CNT-pulp is from the initial neutrality to weak acidity and then raising to alkalinity, The dispersion of tubes in cellulose matrix and characteristics of composite were investigated. Also, electrical resistance for retrieved CNT-based sheets was measured using fore-probe method.
\end{abstract}

Keywords: Carbon Nanotube, Dodecyl Itaconate, Paper-Making

\section{Introduction}

CNTs typify a class of nano-sized materials having many potential applications due to their exceptional structural, mechanical, electronic and thermal properties [1]. It has been demonstrated that the $\mathrm{C}-\mathrm{C}$ covalent bond formed in a graphene layer is one of the most stable chemical bonds, which have made them potentially useful such as nano-reinforced materials, and nanoelecdevices, etc. [2]. However, the inertia of the $\mathrm{C}-\mathrm{C}$ bond also make the as-produced CNTs appear in thick bundles plausible held together by Van der Waals attractions. For every rod-like particle, Van der Waals attractions are highly directional, which favors parallel alignment so that the nano-effectiveness and function of themselves were limited [3]. So far, significant progress has already been established for dispersing CNTs in aqueous and/or organic solvents. In general, two main strategies have been developed to achieve their exfoliation from initial bundles state. One is the so-called chemical modification, which was achieved by functionalizing the surface of tube's sidewall, followed by reaction with chemical group such as fluorinc, alkanes, or diazonium salts [4]. Although the desirable CNT dispersion could be achieved; it deteriorates their inherent mechanical and electrical properties, due to tubes to be shorted aroused simultaneously. Another is the use of some particular chemicals as the dispersant which is well-known the so-called noncovalent functionalization, achieved based on physical adsorption of surfactants or polymers on the tube surface [5]. CNTs obtained in this manner remain their pristine structure/ properties and therefore practicable for applications.

Herein, it is reported a novel-type surfactant, dodecyl itaconate, which is synthesized in one-pot by the present authors. The final reaction product was characterized and the capability for dispersing ability in water was investigated. Furthermore, retrieved aqueous mono-dispersed CNTs with pulp to produce the CNT-based paper were prepared. Different from the reported papermaking process, a novel adjusting $\mathrm{PH}$ process was used to produce paper loaded with CNT in this work.

\section{Experimental}

\subsection{Synthesis of Dodecyl Itaconate as CNTs Disperser}

Dodecyl itaconate was synthesized by a procedure as 
described in Figure 1 [6]. In a typical run for synthesis of DI, the dodecanol $(8.00 \mathrm{~g}, 0.043 \mathrm{~mol})$ was dissolved preliminarily by heating at $50^{\circ} \mathrm{C}$ for $0.5 \mathrm{~h}$, and then itaconic acid anhydride $(5.01 \mathrm{~g}, 0.044 \mathrm{mmol})$ was added. The mixture was stirred at $115^{\circ} \mathrm{C}$ for $2 \mathrm{~h}$ in the nitrogen atmosphere to obtain the dodecyl itaconate. After the reaction was completed, $300 \mathrm{ml}$ hexane was added into the reaction mixture at $80^{\circ} \mathrm{C}$ under a vigorous mechanical stirring, and the crude product of white crystal was precipitated. The crude product was isolated and purified by recrystallized in $500 \mathrm{ml}$ ethanol twice, and followed to vacuum-dry at room temperature for $24 \mathrm{~h}$. The final product was proven to be more than $99 \%$ purity with a melting point $76-78^{\circ} \mathrm{C}$. The yield is between $85-90 \mathrm{wt} \%$ (dodecanol based). The final reaction product was characterized by ${ }^{1} \mathrm{H}$ NMR and FT-IR techniques. ${ }^{1} \mathrm{H}$ NMR spectra were recorded on a JEOL 400 NMR spectrometer operating at $400 \mathrm{MHz}$ for the proton investigation in $\mathrm{CDCl}_{3}$ solution with tetramethylsilane as the internal standard at room temperature.

\subsection{Preparation of Mono-Dispersed Aqueous CNTs}

Dodecyl itaconate was neutralized by sodium hydroxide and $\mathrm{N}, \mathrm{N}$-dimethylbenzylamine at $50^{\circ} \mathrm{C}$ to obtained the corresponding salts. In order to retrieve CNT dispersion aqueous, multi-wall CNTs (MWNTs) supplied by Mitsui-bussan Nanotech Co. Ltd. (Japan) were employed in this study. The raw CNT synthesized by the chemical vapor deposition (CVD) process had the average diameter of 10-30 nm and the length of 5-15 um. For a typical sample, $10.0 \mathrm{~g}$ MWNTs were mixed with $500 \mathrm{ml}$ aqueous loaded with $2.0 \mathrm{~g}$ the above-mentioned SDI, which is solved into water preliminary. The mixture solution was grounded by Biz-miller (DYNO-Mill, Shinmaru Enterprises Corporation) for $2.0 \mathrm{~h}$ at room temperature. In addition, typical scanning electron microscope (SEM) images performed with a JEOL JSM-5610 HV with an electron voltage of $15 \mathrm{kV}$ and a secondary electron detector was used to evaluate the dispersion effect of retrieved aqueous MWNT solution after dilution to 0.001 $\mathrm{mg} / \mathrm{ml}$. The nanoparticle size of CNT in the mono-dispersion aqueous was examined by Dynamic Light Scattering (DLS) measurement, which is performed on a Microtrac HRA 9320-X100 with a measure range as $0.01-1000 \mathrm{~m}$ at room temperature, and fluid and particle<smiles>C=C(CC(=O)OCCCCCCCCCC)C(=O)O</smiles>

Figure 1. Synthetic procedure for DI. refractive index were defined as 1.33 and 2.42, respectively. Meanwhile, ionic surfactant, sodium dodecyl sulfonate (SDS) was employed as a reference to evaluate of competing stabilization characteristics of DI [7].

\subsection{Manufacture of Paper Loaded with CNTs}

After the mechanically mixing the aqueous CNTs and cellulose, poly aluminum chloride and ammonia water were employed to modify $\mathrm{PH}$ of the suspension solution. As a typical acid papermaking process, $\mathrm{PH}$ was adjusted from the initial neutrality to weak acidity $(\mathrm{PH}=6.0 \pm 0.2)$ and then raising to alkalinity ( $\mathrm{PH}=10.0 \pm 0.2)$. In order to compare the properties of paper loaded CNTs, the traditional papermaking process was also prepared depending on adjustment of $\mathrm{PH}$ to mixture suspension solution from the neutrality to alkalinity $(\mathrm{PH}=10.0 \pm 0.2)$ directly. CNTs dispersion effect on single cellulose fiber was observed by SEM, and the electrical resistance was measured using a four-probe method to each sample.

\section{Results and Discussion}

When dodecanol reacted with itaconic acid anhydride to form dodecyl itaconate, the signal peak at $3.64 \mathrm{ppm}$, which ascribed to methylene group of $-\mathrm{CH}_{2}-\mathrm{O}-$ of dodecanol as shown in Figure 2(a), was shift to $4.12 \mathrm{ppm}$, because the hydroxyl group disappeared to substitute with a different chemical group. In Figure 2(b), three novel proton resonances were appeared in the synthesized product, simultaneously. Although the proton

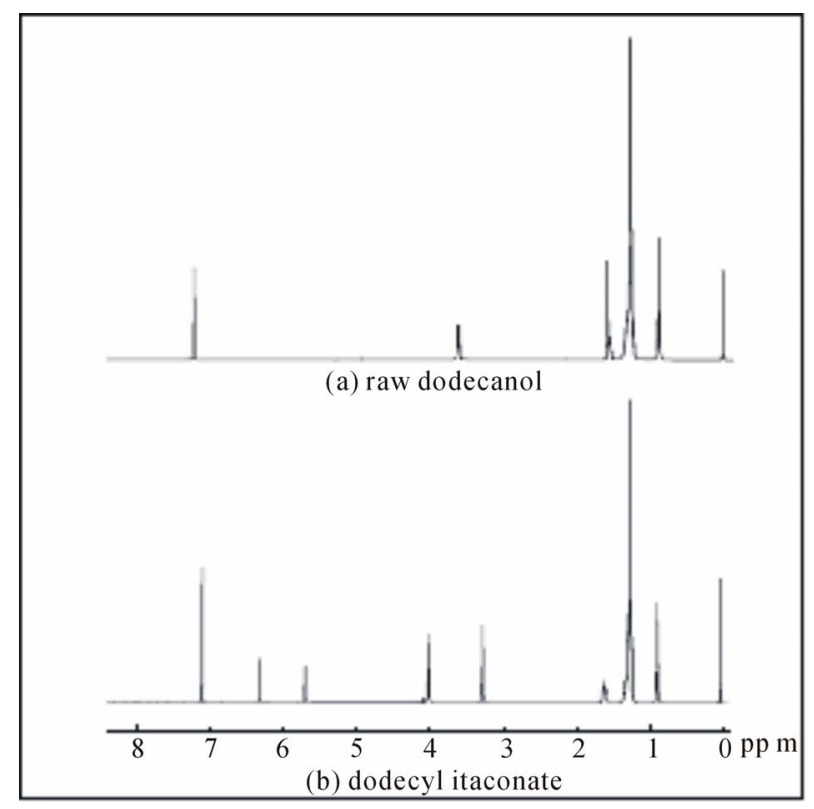

Figure 2. ${ }^{1} \mathrm{H}$ NMR spectra of (a) raw 1-dodecanol, (b) synthesized dodecyl itaconate. (in solvent $\mathrm{CDCl}_{3}$ ). 
resonances from the double bond of the raw itaconic acid anhydride are measured at 6.57 and $5.92 \mathrm{ppm}$ (data is not shown), respectively, it is observed that their signals shift to 6.45 and $5.82 \mathrm{ppm}$ after the final reaction. These results indicated that the ring of acid anhydride was opened and the proton of the double bond was attractive by different adjacent methylene group after reaction. Furthermore, the proton resonance at $3.64 \mathrm{ppm}$, which is corresponding to $-\mathrm{C}(=\mathrm{C})-\mathrm{CH}_{2}-\mathrm{C}(\mathrm{O})$ - of pristine acid anhydride was seen a shift to $3.37 \mathrm{ppm}$. It could be considered an influence from the adjacent ester group. On the other hand, FT-IR results gave the further evidence for the synthetic product to indicate that the peak corresponding to the $\mathrm{C}=\mathrm{C}$ double bond stretching vibration at about $1630 \mathrm{~cm}^{-1}$ appeared in the dodecyl itaconate compared with raw dodecanol material. And, the stretching vibration of the carbonyl groups at about 1685 and $1710 \mathrm{~cm}^{-1}$ merged in the final product.

According to the literature, the adsorption of surfacetant onto CNT solid surfaces usually depends on the characteristics of the tube and nature of the surfactant molecule [8]. DI is a typical linear surfactant, possessing a hydrophobic, C12-alkyl group and a hydrophilic headgroup with an olefin functional group. The initial interaction between surfactant molecules and tubes lie in the parallel the adsorption on side-walls of tubes driven by the hydrophobic interactions exclusively. In this connection, the power from the external matrices is essential to peel individualized tubes from the out-layer of their bundles while surfactant molecule attached onto the CNT surface and then introduce the steric repulsion forces to overcome the interaction between the tube comrades' attractions aroused by Van der Waals. Consequently, a stable mono-dispersion of individual was retrieved, as presented in Figure 3.

Different from the reported surfactant, an olefinic bond functional group was near the head-group for the novel surfactant. It is proposed that the double bond on the DI molecule played an important role to achieve the mono-dispersion of CNTs in water. For verification, we selected cyclic methylsuccinic anhydride to substitute itaconic anhydride for the synthesis of dodecyl methylsuccinic which have a similar chemical structure as DI but without double bond, and dispersed the MWNT, according to above-mentioned procedure. As a result, CNTs dispersed via the dodecyl methylsuccinic aqueous solution was still aggregated seriously. It helps to propose that a monolayer of adsorbed surfactant with the anionic head-groups directed outward to the aqueous phase and the adsorption reaches equilibrium depending to function of the olefin bond on the DI molecules. On the other hand, as listed in Table 1, the results of nanoparticle size distribution obtained by DLS meas- urement were $\sim 4.1 \mathrm{~m}$ and $\sim 5.8 \mathrm{~m}$ in an average volume for DI and SDS surfactant, respectively, and approximate with their SEM observation. This result is also proved mono-dispersion state of dispersed solution as indicated SEM imagines. It has been reported that CNTs solubilized by SDS could be preserved as the soluble state more than $500 \mathrm{~h}$ at room temperature [9]. In order to explore the characteristic of DI, a heating treatment was used in this research work. It is known that the colloidal stability in the surfactant solution will be destroyed dur- ing thermal treatment, because of the inner molecular activity. Although both of aqueous CNT solubilization presented a mono-dispersion from their aggregated bundles, individual MWNTs exfoliated by SDS gave a re-aggregation after the thermal treating at $50^{\circ} \mathrm{C}$ for $168 \mathrm{~h}$ as indicated in Table 1. It could be explained that $\pi$ - electron of the double bond functional group in DI structure provide the colloidal stability. In fact, $\pi-\pi$ stacking such as benzene rings onto the surface of graph- ite is reported because it could be considered that func- tion could increase the binding and coverage of surface- tant molecules to graphite significantly [10]. According to our knowledge, this is the first report to examine function of double bond on surfactant molecules for the CNT dispersion in the water.

In order to improve the CNT/cellulose paper, acid papermaking process was employed in this work. Figure 4
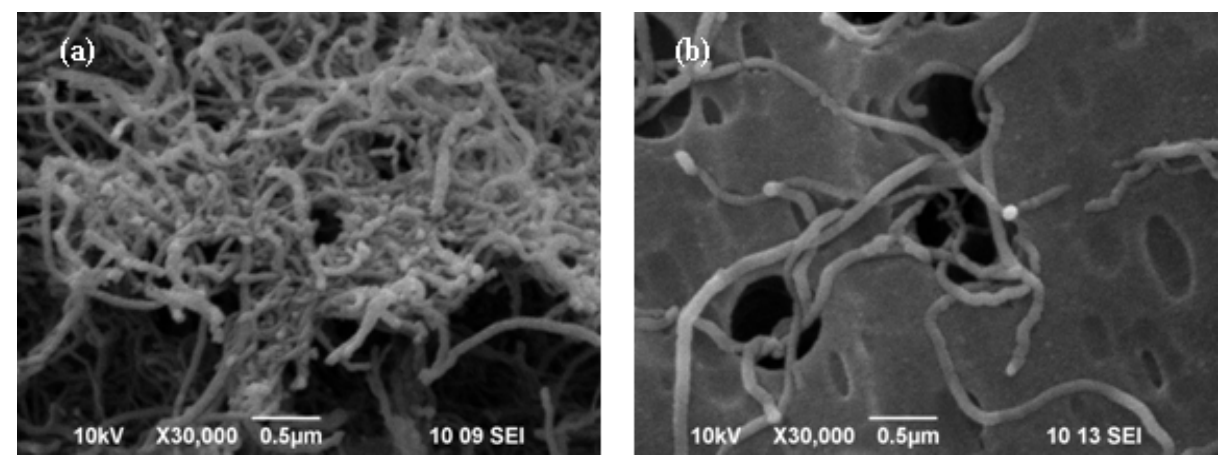

Figure 3. SEM micrographs of dispersed CNT (a) without and (b) with DI. 
Table 1. DLS measurement results or dispersed CNT.

\begin{tabular}{|c|c|c|c|c|}
\hline Surfactant & Treatment & $\operatorname{Mv}(\mathrm{m})^{1}$ & $\operatorname{Mn}(\mathrm{m})^{2}$ & $\mathrm{Ma}(\mathrm{m})^{3}$ \\
\hline \multirow[t]{2}{*}{ DI } & r.t. & 4.119 & 2.884 & 3.710 \\
\hline & $50^{\circ} \mathrm{C} / 168 \mathrm{~h}$ & 5.868 & 3.065 & 5.309 \\
\hline \multirow[t]{2}{*}{ SDS } & r.t. & 5.868 & 5.319 & 5.309 \\
\hline & $50^{\circ} \mathrm{C} / 168 \mathrm{~h}$ & 15.710 & 8.897 & 12.880 \\
\hline
\end{tabular}

1. Mv: average volume, 2. Mn: average number, 3. Ma: average area.
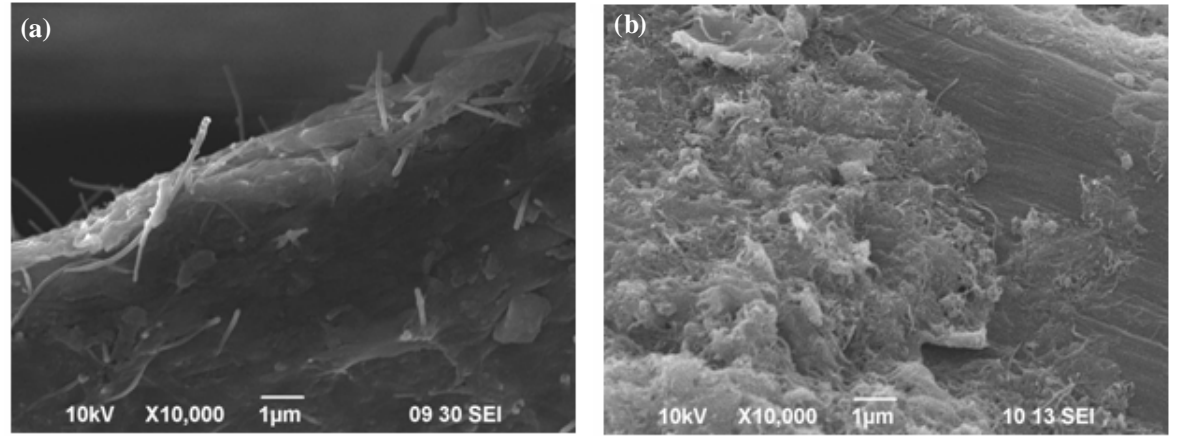

Figure 4. SEM micrographs of dispersed CNTs on single cellulose fiber (a) in the acid and (b) in the general papermaking.

shown the interconnected network established by the individual tubes on the surface of cellulose fiber in the $\mathrm{CNT} /$ cellulose paper, and its good conductivity was also presented, simultaneously. It is well known that the form of CNT network in (on) to the matrices would help the conductivity development of CNT. In this work, the objective is achieved by preparation of mono-dispersed CNTs aqueous and followed by transferring these tubes onto the surface of cellulose fibers by the PH adjustment to acidity for the CNT-pulp suspension. Although the mechanism for acid papermaking process is being under studied at present, it is reasonable to consider that the acidity could influence the mono-dispersion of CNTs because some DI salt would be reduced to the DI resulting to the homogeneous individual tubes interacted with cellulose fiber and formed corresponding CNT network [11]. On the other hand, the vigorous morphological change on the surface of the tubes was shown the agglomeration for the general papermaking process. The result also certified that the $\mathrm{PH}$ adjustment in the papermaking play an important role to transfer the individual CNT tubes from the aqueous solution to the cellulose fibers, forming a "CNT-pulp". Furthermore, it should be emphasized the CNT effectiveness that the electrical resistance was increased with loaded CNTs amount to both of papermaking process, as shown in Figure 5. For the expensive CNT material, what net-work structure was formed onto surface of cellulose fibers would be more practical to retrieve higher properties CNT-paper. Additionally, polymerizable capacity of DI functional-

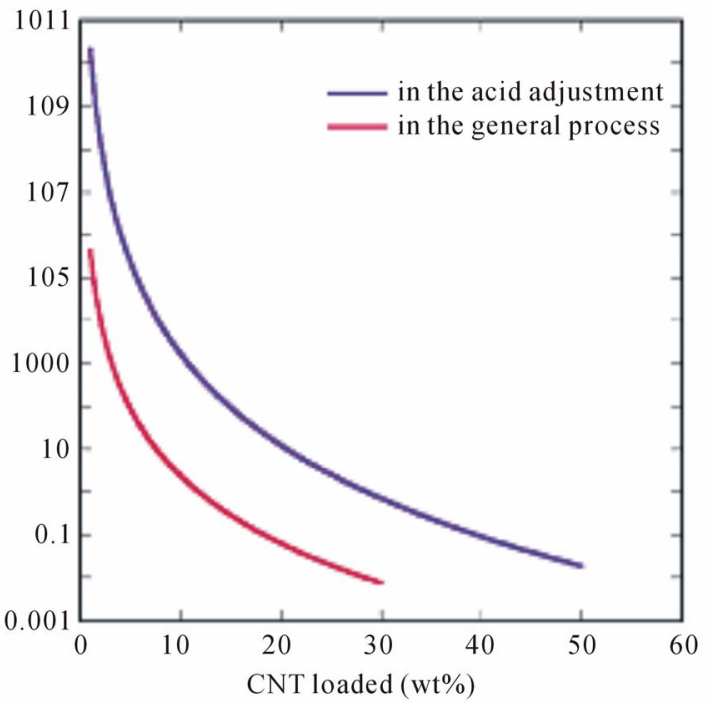

Figure 5. Electrical resistance for CNT-based paper.

ized as a monomer is expected to trigger the further research due to high reactivity of olefinic carbon. At present, wrapping ultrathin polymer film formed by DI polymerization on CNTs surface to diversify their application in the other nano-composites field is being processed.

\section{Summary}

In summary, we have demonstrated a simple procedure for a novel-type surfactant DI in one-pot. Due to $\pi$-elec- 
tron function of double bond group on the surfactant molecule, aqueous mono-disperse CNTs was retrieved. Also, it provides the mono-dispersion solution to keep colloidal stability for longer period than general surfacetant. Different from the general papermaking process, a novel adjusting $\mathrm{PH}$ process was used to preparation the paper loaded with CNT. As a result, this method could transfer easily dispersing mono-dispersed CNTs in aqueous onto the surfaces of cellulose fibers homogenously and higher properties CNT-paper was retrieved.

\section{Acknowledgement}

This work was supported by funding from Grants-in-Aid for Scientific Research from the Ministry of Education, Culture, Sports, Science and Technology of Japan (No. 18310049), by the Japan Science and Technology Agency (JST), and by the Ministry of Health, Labor, and Welfare (No. H18-Chem-Gen-006).

\section{References}

[1] S. Iijima, "Helical Microtubules of Graphitic Carbon," Nature, Vol. 354, 1991, pp. 56-58. doi:10.1038/354056a0

[2] S. Frank, P. Poncharal, Z. L. Wang and W. A. de Heer, "Carbon Nanotube Quantum Resistors," Science, Vol. 280, No. 5370, June 1998, pp. 1744-1746. doi:10.1126/ science.280.5370.1744

[3] L. A. Girifalco, M. Hodak and R. S. Lee, "Carbon Nanotubes, Buckyballs, Ropes, and a Universal Graphitic Potential," Physical ReView B, Vol. 62, No. 19, 2000, pp. 13104-13110. doi:10.1103/PhysRevB.62.13104

[4] D. Tasis, N. Tagmatarchis, V. Georgakilas and M. Prato, "Soluble Carbon Nanotubes," Chemistry - A European Journal, Vol. 9, No. 17, September 2003, pp. 4000-4008. doi:10.1002/chem. 200304800

[5] O. Matarredona, H. Rhoads, Z. Li, J. H. Harwell, L. Balzano and D. E. Resasco, "Dispersion of Single-Walled Carbon Nanotubes in Aqueous Solutions of the Anionic Surfactant NaDDBS," Journal of Physical Chemistry B, Vol. 107, No. 48, November 2003, pp. 13357-13367. doi: 10.1021/jp0365099

[6] J. Ozawa, G. Matsuo, N. Kamo and K. Tsujii, "Separated Organized Polymerization of an Amphiphilic Monomer and Acrylamide in One-Pot Reaction," Macromolecules, Vol. 39, No. 23, October 2006, pp. 7998-8002. doi:10. $1021 / \mathrm{ma} 0611321$

[7] B. Vigolo, C. Coulon, M. Maugey, C. Zakri and P. Poulin, "An Experimental Approach to the Percolation of Sticky Nanotubes," Science, Vol. 309, No. 5736, August 2005, pp. 920-923. doi:10.1126/science.1112835

[8] J. Djuve, L. M. Grant, J. Sjoblom, T. P. Goulob and R. J. Pugh, "Templating of Ethyl(hydroxyethyl)Cellulose on Graphite by Surfactant-Polymer Interactions," Langmuir, 2002, Vol. 18, No.7, 2002, pp. 2673-2677.

[9] B. Zhao, H. Hu, A. Yu, D. Perea and R. C. Haddon, "Synthesis and Characterization of Water Soluble Single-Walled Carbon Nanotube Graft Copolymers," Journal of the American Chemical Society, Vol. 127, No. 22, May 2005, pp. 8197-8203. doi:10.1021/ja042924i

[10] M. F. Islam, E. Rojas, D. M. Bergey, A. T. Johnson and A. G. Yodh, "High Weight Fraction Surfactant Solubilization of Single-Wall Carbon Nanotubes in Wate," Nano Letters, Vol. 3, 2003, pp. 269-273. doi:10.1021/n1025 $924 \mathrm{u}$

[11] B. Fugetsu, E. Sano, M. Sunada, Y. Sambongi, T. Shibuya, X. S. Wang and T. Hiraki, "Electrical Conductivity and Electromagnetic Interference Shielding Efficiency of Carbon Nanotube/Cellulose Composite Paper," Carbon, Vol. 46, No. 9, 2008, pp. 1256-1258. doi:10.1016/j.carbon.2008.04.024 